\title{
Possible Causes of Mentalization Deficiency and Later Diagnosed Borderline Personality Disorder: A Systematic Review
}

\author{
Bonica Wang ${ }^{1, \mathrm{a}}{ }^{\dagger}$, Sophie $\mathrm{Xiao}^{2, \mathrm{~b} \dagger}$, Helena Wang ${ }^{3, \mathrm{c} \dagger}$ \\ ${ }^{1}$ College of Art and Science, New York University, New York, United States \\ ${ }^{2}$ Division of Science and Technology, Beijing Normal University - Hong Kong Baptist University United \\ International College, Guangdong, China \\ ${ }^{3}$ Tulane School of Liberal Arts, Tulane University, New Orleans, United States \\ ajw5558@nyu.edu, ${ }^{b} k 530016014 @$ alumini.uic.edu.cn, ${ }^{c} r w a n g 19 @ t u l a n e . e d u$ \\ These authors contributed equally.
}

\begin{abstract}
Mentalization is the capability of individuals to understand the mental states of themselves and others. Patients with Borderline Personality Disorder (BPD) are characterized by deficient mentalization abilities, which result in impaired social functioning and distorted self-identities. With the acknowledgement of the importance of mentalization for BPD patients, this review aims to summarize the possible causes of mentalization deficiency among BPD patients. A systematic review is conducted using EBSCO Discovery Service (EDS), EBSCOhost APA PsycARTICLES, and Google Scholar for the period between 2010 and 2020. The descriptors used are 'borderline personality disorder' and 'mentalization'. From the neurological aspects, when performing social cognition tasks, BPD patients display the usage of a different brain pathway characterized by the hyperactivation in amygdala and hypoactivation in brain areas related with Theory of Mind. From the genetic aspects, certain gene alleles are possible determinants for the impaired mentalization abilities. From the psychological aspects, the mentalization deficiency is affected by early childhood experiences, especially abuses. These result in the later diagnosed borderline personality disorder and damage-related psychological functions. It is concluded that the mentalization deficit among BPD patients can be traced back to genetics, neurological causes, early childhood experience, and psychological causes. And these findings may contribute to the diagnosis, treatment, and early prevention of Borderline Personality Disorder.
\end{abstract}

\section{Keywords: Borderline Personality Disorder, Mentalization}

\section{INTRODUCTION}

Borderline personality disorder (BPD) is a common mental disorder. BPD patients usually have unstable relationships, self-images, and emotions. The significant features of the disorder include emotional dysregulation, high levels of impulsivity which can easily cause selfharm, and disturbed interpersonal functioning which leads to high levels of preoccupied and disorganized attachment patterns [1]. As for the mentalization-based approach to BPD, patients show dissociative comorbidity, which is related to the emotional neglect and stress [1]. People with BPD have disorganization of self-structure that can cause splitting thinking. This "white and black thinking" means that they are not able to integrate the positive and negative opinions of themselves and others.
BPD patients with a lifetime prevalence are estimated to be $6 \%$ in the general population [1]. BPD patients can be traumatized due to several trivial matters. Women are more likely to be afflicted by BPD than men. Men with BPD tend to have drug abuse, while women with BPD tend to have eating disorders. The dysfunctional attachment patterns, the neglectful and abusive nurturing environments, and the genetic factors can all lead to BPD.

Mentalization refers to' the ability of individuals to understand and interpret the meaning of the behaviors of their own or others via considering underlying mental states [2]. Fonagy(1991) points out that BPD patients have the unstable capability for mentalization [1]. Three modes of thinking disturb individuals to acquire robust mentalization, including psychic equivalence mode, pretend mode, and teleological stance mode [3]. BPD 
patients with the thinking of psychic equivalence mode tend to equate their inner state with external reality. It means that psychic equivalence mode impedes people to acquire alternative perspectives on reality. People with psychic equivalence mode are more likely to develop the temporal paranoid ideas, and their inner states become quite real. The pretend mode refers to the decoupling of mind from reality, which is the opposite to the psychic equivalence mode. BPD patients may find their ideas unmodulated by reality [3].

When children are able to integrate the previous two modes, they realize that their inner minds are linked but separate from the external reality and thus develop mentalization. The third thinking mode is the teleological stance, which means that people acknowledge the existence of mental state only when they observe the existence of external physical events. In this case, people focus more on actions but less on ideas or words [3].

When people lack stable mentalization, they are unable to handle negative thoughts and extreme emotions. BPD patients who have insufficient mentalization do not have the capabilities to complete self-regulation, and they cannot precisely realize the mental states of others when they are in intimate relationships. As for BPD children, their attachment patterns are activated at the wrong time which disturbs their mentalization development.

There are several useful methods to measure the levels of mentalization of individuals, including selfreports, reflective-function (RF) construct, and the Revised Reading the Mind in the Eyes Test (RET). Selfreports that measure adults' attachment patterns reveal that BPD patients have fearful and preoccupied insecure attachment patterns. RET, which refers to receiving the mind in the eyes test, measures how well the participant can put himself/herself into the mind of the other person [4]. It tests individuals' capability to recognize others' subtle emotional states. The Reflective Function (RF) construct tests people's awareness of the mental states in others and themselves, as well as the mutual influences at work between mental states and behavior [4].

This paper aims to study the possible causes of mentalization deficiency among BPD patients. The causes of mentalization deficiency from genetic aspects and psychological aspects are analyzed.

\section{METHOD}

In developing this research, a systematic search has been conducted using Google Scholar. Several search terms were used and combined such as "BPD" or "Borderline Personality Disorder" or "Borderline Disorder", "mentalization". Also, the paper of the first authors of the articles chosen and the reference page of chosen articles are also examined to find related research. This results in 56 records. The following criteria were used for screening and selection:(1)The article must be published in English and is peer-reviewed, full-text, and available, (2) The result in the article must be empirical and original, (3) The article must use reliable tools to measure borderline personality disorder, (4) The article reports results with respect to mentalization. Articles are excluded if their participants are not human, if they are not written in English, and if they are not related to mentalization and BPD. In total, 20 articles were included in this review.

\section{RESULTS}

Taken together, the causes of mentalization defects include in BDP the following psychological and physical aspects. Next, we will introduce each of them.

\subsection{Neurological Aspects}

With the development of brain imaging techniques, it is much easier for researchers to find out the reasons causing mentalization deficits. A lot of studies have been conducted to investigate aspects like brain activities and brain structures regarding mentalization deficits in BPD patients. Several brain regions, especially the amygdala, mirror neuron system, and regions for ToM, are highlighted as they showed abnormal activities compared with healthy controls.

A few studies have claimed the importance of amygdala activities in BPD patients. Mier et al. who conducted fMRI on BPD patients investigating their social cognition ability found hyperactivation in the amygdala [5]. The fMRI study carried out by Frick et al. also shows a hyperactivation in the amygdala during mindreading tasks in BPD patients [6]. The amygdala is in the limbic system which processes emotional stimuli unconsciously. Activation of the amygdala after seeing a person's face “conveys information about emotional aspects of facial expressions via the insula to the inferior prefrontal cortex where action representation and the recognition of intentions take place" which is the process of emotion simulation [5]. Hyperactivation in the amygdala reveals that BPD patients are much easier to be aroused by the facial stimuli than the healthy controls since the healthy controls didn't show any amygdala activation. Therefore, activation of the amygdala in BPD patients indicates that when involving social cognitive activities, they have difficulties distinguishing emotions of others from themselves, implying mentalization failure.

However, these two studies are not consistent in brain activities in ToM regions. Mier et al. found hypoactivation in areas of the mirror neuron system including superior temporal sulcus and Brodmann Area 44 of the inferior frontal gyrus [5]. The mirror neuron system is essential for the Theory of Mind which helps people carry out social activities by making attributions of mental states to both themselves and others 
Hypoactivation in MNS indicates that while involved in social cognition activities, BPD patients have trouble understanding and inferring others' thoughts, which signals mentalization failure. In contrast, Frick et al. reported that BPD patients also have hyperactivation in the left temporal pole and the middle temporal gyrus [6]. These two brain regions are also recognized as ToM regions indicating BPD patients have a great ability to estimate what others are feeling.

Despite fMRI studies, MRI studies have shown that BPD patients have different brain structures from healthy controls. Boen et al. conducted an MRI study and found a reduced cortical thickness in the medial prefrontal cortex and left temporoparietal junction [7]. These two regions of the brain are linked with ToM which is closely related to mentalization capabilities. A reduced cortical thickness can result in BPD patients having an incomplete mentalization ability, which would lead to an incorrect perception of others and their own identities.

\subsection{Genetic Aspects}

Genetics plays an important role in causing BPD. Studies before 2000 have already demonstrated the inheritability of BPD by using twin studies. Recently, researchers have found specific genotypes that could cause BPD and contribute to metalization deficits.

The protein-coding gene HTR1A has been noticed by Zetzsche et al [8]. After scanning the brain structures of BPD patients, they found that amygdala volume varies based on different alleles of HTR1A gene patients possess. "The volume of the amygdala of patients carrying the $\mathrm{G}$ allele was significantly lower than that of patients with a homozygous $\mathrm{C} / \mathrm{C}$ genotype."[8] Activities in the amygdala in BPD patients have been proved to be essential for mentalization capabilities. And a higher amygdala volume causing possibly higher amygdala activation can lead to BPD patients hypermentalize the social stimuli, which has been proved to be problematic. Hence, possessing $\mathrm{C} / \mathrm{C}$ alleles of the HTR1A gene can be recognized as a possible cause of mentalization deficit.

\subsection{Psychological Aspects}

\section{Abuses}

Abuses have been considered as the main cause of borderline personality disorder. It includes physical abuses, psychological abuses, and neglect. Abuses do harm to people's physical and mental health development, resulting in impaired mentalization and problematic behaviour. Also, the abuse that happened in childhood has long-term effects and leads to borderline personality disorder traits and co-occurring mental disorders [9][10]. In Keng and Wong's study, child abuse has a great predictor and effect on the formation of borderline personality disorder [9].

\subsubsection{Physical Abuses}

Physical abuses are aggressive behaviours that happen on victim's physical bodies, like violent attacks or sexual abuses. It contributes to the childhood invalidation and formation of early borderline personality disorder. Also, borderline personality disorder patients frequently report that they have experienced child abuse [9]. For example, women who have suffered from child abuse or childhood trauma are two times more likely to have a cluster B personality disorder [11]. In BDP, troubling childhood experiences and memories have been considered as the main cause. Mostly they have an unhealthy or bad relationship with family and others. And they also have self-destructive behaviours to express inner anger and manipulation, like showing their aggressive meaning and attack by breaking the counseling settings and acting suicidal [12]. This is a way for borderline personality disorder patients to reflect the trauma that they have suffered from the abuse in childhood. In physical abuse, childhood sexual abuse (CSA) have been considered the most traumatic event, associated with borderline personality disorder [13].

\subsubsection{Psychological \& Neglect}

Psychological abuse includes verbal abuse and emotional maltreatment. And neglect includes abundance, rejection, and parenting absence. Actually, these mostly impact victims' mental levels. In Keng and Wong's study, it illustrates that parental invalidation has significantly and negatively correlated with self-compassion, which is negatively correlated with BDP symptomatology [9].

\subsection{Environmental Aspects}

In Grogan-Kaylor et al. longitudinal study (2020), they found that the physical child abuse can spread across time and space [14]. They have analyzed the data of police-investigated PCA in 15 years. The rate of PCA is associated with the median household income, child care burden, the percentage of the population of a Census tract who were children and ethics. This result indicates that the occurrence of child physical abuse has a close relationship with the community and family circumstances.

\subsection{Individual Aspects}

For individuals, child abuses are traumatic events for victims. The traumatic experience affects their mentalization development. Children who have suffered from CSA will experience anxiety, fear, depression, and anger in the short-term effect, potentially inducing mental problems in adulthood, easily developing sexual dysfunction and self-injury. Being hard to trust other people and establish a stable close relationship are common in this population. Because of the CSA, self- 
esteem is being impaired and the anger of the assailant can not be relieved, in turn, being aggressive and impulsive in some way to indicate their pains. And these symptoms are in accord with the characteristics of BDP, which are also considered precursor symptoms [15]. That means victims are more likely to show the pre-symptom of borderline personality disorder. And the maltreated experience will affect their mentalization

And the reflective functioning is the ability to evaluate the mentalization development, also is the protective role of mentalizing [16, 17]. In contrast, people who suffered from childhood abuse, especially emotional maltreatment, have high rejection sensitivity and more risk to develop borderline personality disorder [17, 18].

People who have suffered from emotional abuse and neglect are more likely to develop high rejection sensitivity and low self-esteem, which directly links to borderline personality disorder [18]. Both physical and psychological abuses are correlated with rejection sensitivity, but the psychological abuses are more significantly related to rejection sensitivity. And in the borderline sample, the emotional abuse and neglect also directly relates to rejection sensitivity. Compared to other mental disorders, borderline patients have a higher level of rejection sensitivity.

In previous studies, scholars have found that reflective functioning plays a mediator role between mentalization and borderline personality disorder [16, 17]. Quek et al. claim that both emotional abuse and neglect are indirectly affecting adolescents' borderline pathology [17]. That means childhood maltreatment affects mentalization through reflective functioning. People who have low levels of reflective functioning are more likely to show borderline personality features. So mentalization-based therapy, which aims to improve reflective functioning, is useful for maltreated victims. It benefits in developing reflective functioning to indirectly affect borderline features. If the level of reflective functioning can be improved, the possibility and risks of borderline personality disorder will be lower [16].

\subsection{Parenting Aspects}

For maltreated parents, the impacts of childhood abuses will pass to their offsprings through parenting. Some research shows that this leads to an intergenerational consequence in which maltreated parents' children are more likely to show prepsychopathological symptoms, like neurobiological anomalies and disorganized attachments [16]. That means the impact of child abuse also affects rising offsprings because maltreated parents cannot be a good model for their kids in mentalization. And their behaviors, like borderline personality symptoms, will affect the growth of kids, becoming an intergenerational trauma.

\section{DISCUSSION}

Possible causes of mentalization deficiency in BPD patients are categorized into three main aspects which are neurological, genetic, and psychological. They contribute to the impaired mentalization capability in BPD patients in different ways.

\subsection{Neurological Aspects}

As the result shows, many studies have connected the amygdala with mentalization abilities as BPD patients would exhibit hyperactivation in the amygdala during social cognition tests. Amygdala has been identified as the brain region that is involved in "emotion simulation" after seeing emotional stimuli [5]. Therefore, the hyperactivation in the amygdala signals that BPD patients process the information on others' faces more emotionally than the healthy controls, meaning that they would also experience the emotion of the stimulus' rather than simply interpret the takeaway message from it. Hence, with higher amygdala activation, BPD patients will have difficulties distinguishing the feelings of oneself from the others', causing a distortion in their own identity recognition and social cognition. It can also explain why BPD patients are usually withdrawn from society. Amygdala hyperactivation during social cognition would result in BPD patients more inclined to notice the negative stimuli and produce feelings of being threatened because they are simulating the same negative stimuli themselves [5]. Thereby, negative stimuli causing negative interpretation form a vicious cycle that stops BPD patients from involving themselves in social activities.

Apart from amygdala hyperactivation, hyperactivation in mirror neuron regions is also detected in several studies, which back up the theory of "emotion simulation". The mirror neuron region is often activated when people imitate others' actions in their brain. Hyperactivation in mirror neurons during the social cognition process also indicates that BPD patients are resonating with the stimuli they received [6]. However, it is a sign of mentalization deficit because resonating is not required during basic social cognition like guessing intentions of others. Hyperactivation in both amygdala and mirror neurons shows that BPD patients have distorted self-identity since there is a very vague bond between BPD patients' own emotions and others' emotions during social interaction. This mentalization deficit has caused BPD patients to be emotionally active in both understanding the emotion and simulating the emotion.

Moreover, the hypoactivation in Theory of Mind regions shows that $\mathrm{BPD}$ patients lack conscious reflection when involved in social cognition. Their abilities to simulate and understand the message behind the facial expression are excessively functioned while the 
process of making a rational judgement regarding potential intention is defective. Moreover, the prefrontal cortex is responsible for higher-order thinking and processing which includes the perception of self-identity. A lack of activation in such regions reflects BPD patients' inability to produce a concrete perception of their own identities, which is also a form of mentalization deficit.

Therefore, from the neurological patterns, it can be concluded that BPD patients use an alternative brain pathway when processing social information. It is the exact brain pathway they use that causes their incomplete mentalization and impedes their normal social functioning. Hence, it gives psychologists and therapists hints upon the suitable treatment for BPD.

Despite the difference in brain activation, some studies also showed a thinner brain cortex in BPD patients' brains which might be the cause for mentalization deficits. A thinner brain cortex can be formed due to several reasons. It is possible that BPD patients contain certain genes that restraint brain development in areas related to mentalization, and thus cause deficits. It is also possible that certain childhood experiences make the brain regions related to mentalization not trained enough so that due to neuroplasticity, these brain regions result in a thinner cortex. Therefore, understanding the genetic components and external experiences of BPD patients is very critical for development.

\subsection{Genetic Aspects}

Genetical research has revealed the heritable nature of Borderline Disorder and its impact on mentalization. The detection of HTR1A alleles has pointed out the importance of genetics in determining the capacity of one's mentalization skills.

Therefore, by understanding more about gene fragments, it would be easier for psychologists to identify the population who have higher risks of developing BPD at a very young age. With early precaution, it is possible to help prevent BPD formation and thus decrease the amount of BPD patients.

\subsection{Psychological Aspects}

From the psychological aspect, the main cause of borderline personality disorder is different kinds of abuses, especially childhood abuse. These abuses have a long-term effect on victims which raises the possibility of BPD diagnosis and other BPD symptoms in later adulthood. Besides negative impacts on individuals, these traumas also can be an intergenerational issue, passing onto victims' offsprings through problematic parenting. Also, violent behaviours are easily being spread among communities, raising the number of family abuse cases. That means more children will be at risk of childhood abuse.

\subsection{Clinical Application}

As mentalization is an important factor for BPD, mentalization-based therapy(MBT) has become one of the effective therapies. In order to improve BPD patients' related functions, MBT has some techniques to work with their patients. In an 8-year longitudinal study, Bateman and Fonagy have found that patients who have received MBT are fewer suicide attempts, less demanding of psychoactive medications and emergency rooms [19]. MBT group has shown better performance and long-term effect. In the comparison between MBT and supportive psychotherapy, researchers have found that both of them have significant effects on the treatment for BPD patients. But MBT has higher and better outcomes from patients' self-reports [20]. The importance and influence of mentalization in treatments for BPD patients have been supported. So research of mentalization in the BPD field is necessary and meaningful.

This review is crucial because it puts together the possible causes of mentalization deficiency from all perspectives. With a thorough understanding of these possible causes and effects, making the diagnosis of BPD will become easier. Moreover, since the importance of mentalization in BPD patients has been highlighted, treatment methods like improving the mentalization ability can be proved to be effective measures to help BPD patients relieve their symptoms. In addition, the possible psychological and genetic causes of mentalization deficiency make it possible to help prevent BPD at an early stage.

The limitation of the current review is that the impact of abuses can not be separated, because most of the abuses are including physical attacks and psychological insults. However, which kinds of abuse is the most traumatic for the patients, contributing to borderline personality disorder, still needs further study. And the mentalization-based treatment also can not totally repair patients' injured mentalization. The effective treatment for BPD also needs to be developed. Moreover, there are a limited amount of studies that have been reviewed in this paper. If more studies with respect to BPD and mentalization can be included, this review will be more thorough and convincing.

\section{CONCLUSION}

This review summarizes the possible causes for mentalization deficiency and their impacts with regards to biological and psychological aspects. For biological aspects, BPD patients are using different brain pathways which focus more on emotion simulation rather than intention interpretation. This over-mentalization would 
cause BPD patients to be more sensitive to the negative stimuli in the social environment and would withdraw them from future social activities. Also, the reason for the usage of a different brain pathway and the structural difference can be provoked by the environmental factors like different attachment styles and abuse. In addition, it can also be attributed to genetics. Therefore, if further studies can study these aspects with neuroimaging techniques, researchers would have a more thorough understanding of impacts of nature and nurture on BPD.As for the psychological aspects, abuses impact the development of people's mentalization, resulting in the impaired reflective function and low self-esteem, and victims are showing the early-BPD symptoms. Therefore, these victims are at high risk of later diagnosed BPD.

\section{REFERENCES}

[1] P. Fonagy, P. Luyten, L. Strathearn, Borderline personality disorder, mentalization, and the neurobiology of attachment. Infant Mental Health Journal, 2011, pp. 47-69. DOI: https://doi.org/10.1002/imhj.20283

[2] J. Quek, G. Melvin, C. Bennett, et al., Mentalization in Adolescents With Borderline Personality Disorder: A Comparison With Healthy Controls. Journal of Personality Disorders, 2019, pp.145-163. DOI: https://doi.org/10.1521/pedi_2018_32_336

[3] L. W. Choi-Kain, J. G. Gunderson, Mentalization: Ontogeny, Assessment, and Application in the Treatment of Borderline Personality Disorder. American Journal of Psychiatry, 2008, pp. 11271135.

DOI: https://doi.org/10.1176/appi.ajp.2008.07081360

[4] A. Fossati, J. Feeney, C. Maffei, S. Borroni, Thinking about feelings: Affective state mentalization, attachment styles, and borderline personality disorder features among Italian nonclinical adolescents, Psychoanalytic Psychology, 2014, pp. 41-67. DOI: https://doi.org/10.1037/a0033960

[5] D. Mier, S. Lis, C. Esslinger, et al., Neuronal correlates of social cognition in borderline personality disorder, SCAN, 2013, pp. 531-537, DOI: $10.1093 /$ scan/nss028

[6] C. Frick, S. Lang, B. Kotchoubey, et al., Hypersensitivity in Borderline Personality Disorder during Mindreading, PLoS ONE, 2012, e41650. DOI: 10.1371/journal.pone.0041650

[7] E. Bøen, L. T. Westlye, T. Elvsashagen, et al., Regional cortical thinning may be a biological marker for borderline personality disorder, Acta Psychiatr Scand, 2014, pp. 193-204. DOI: 10.1111/acps. 122
[8] A. Amad, N. Ramoz, P. Thomas, R. Jardri, P. Gorwood, Genetics of borderline personality disorder: Systematic review and proposal of an integrative model, Neuroscience and Biobehavioral Reviews, 2014, pp. 6-19. DOI: http://dx.doi.org/10.1016/j.neubiorev.2014.01.003

[9] S.L. Keng, Y.Y. Wong, Association among selfcompassion, childhood invalidation, and borderline personality disorder symptomatology in a Singaporean sample. Borderline Personality Disorder and Emotion Dysregulation, 2017, pp. 1-8. DOI: https://doi.org/10.1186/s40479-017-0075-3

[10] Á. Frías, C. Palma, Comorbidity between posttraumatic stress disorder and borderline personality disorder: a review. Psychopathology, 2014, pp. 1-10. DOI: https://doi.org/10.1159/000363145

[11] J. C'de Baca, D.T. Castillo, J. E. Mackaronis, C. Qualls, Ethnic Differences in Personality Disorder Patterns among Women Veterans Diagnosed with PTSD. Behavioral Sciences (Basel), 2014, pp. 72 86. DOI: https://doi.org/10.3390/bs4010072

[12] J.S. Nevid, S.A. Rathus, B. Greene, Abnormal psychology in a changing world, Person

[13] A. Barrett, Y. Kamiya, V.O. Sullivan, Childhood sexual abuse and later-life economic consequences. Journal of Behavioral and Experimental Economics, 2014, pp.10-16. DOI: https://doi.org/10.1016/j.socec.2014.07.001

[14] A. Grogan-Kaylor, J. Ma, S.J. Lee, S. Klein, A longitudinal analysis of the spatial spread of policeinvestigated physical child abuse. Child Abuse \& Neglect, 2020, pp. 1-10. DOI: https://doi.org/10.1016/j.chiabu.2019.104264

[15] N.R. Crick, D. Murray-Close, K. Woods, Borderline personality features in childhood: a short-term longitudinal study, Development and Psychopathology, 2005, pp. 1051-1070. DOI: https://doi.org/10.1017/S0954579405050492

[16] B. Nicolas, L. Roxanne, G. Julia, et al., The protective role of mentalizing: reflective functioning as a mediator between child maltreatment, psychopathology and parental attitude in expecting parents, Child Abuse \& Neglect, 2019, pp. 1-10. DOI: https://doi.org/10.1016/j.chiabu.2019.104065

[17] J. Quek, L. Newman, C. Bennett, et al., Reflective function mediates the relationship between emotional maltreatment and borderline pathology in adolescents: a preliminary investigation. Child Abuse \& Neglect, 2017, pp. 215-226. DOI: https://doi.org/10.1016/j.chiabu.2017.08.008 
[18] M. Foxhall, C. Hamilton-Giachritsis, K. Button, The link between rejection sensitivity and borderline personality disorder: a systematic review and metaanalysis, The British Psychological Society, 2019, pp.289-326. DOI: 10.1111/bjc. 12216

[19] A. Bateman, P. Fonagy, 8-year follow-up of patients treated for borderline personality disorder: mentalization-based treatment versus treatment as usual, The American journal of psychiatry, 2008, pp. 631-638.

DOI: https://doi.org/10.1176/appi.ajp.2007.07040636

[20] C.R. Jørgensen, C. Freund, R. Bøye, et al., Outcome of mentalization-based and supportive psychotherapy in patients with borderline personality disorder: A randomized trial, Acta Psychiatrica Scandinavica, 2013, pp.305-317. DOI: https://doi.org/10.1111/j.1600-0447.2012.01923.x 